\title{
Characteristics of the behavior of a liquid film in a pulsed gas-droplet flow
}

\author{
Pavel Karpov ${ }^{1, *}$, Nikolay Miskiv ${ }^{1}$, Anatoly Serov ${ }^{1}$ \\ ${ }^{1}$ Kutateladze Institute of Thermophysics SB RAS, Lavrentiev av. 1, Novosibirsk, 630090, Russia
}

\begin{abstract}
The results of the investigation of the influence of a multi-jet impact air flow on the process of formation of a liquid film from a drop flow on the vertical surface of a heat exchanger with a low temperature are presented. Characteristics of the film flow and the effect of this flow on heat transfer, shown in the paper, are related to the interaction of the boundary layer of the wall air flow and the perturbation induced by the droplets under the pulsed regime of introducing the cooling mass into the flow of impact air jets. The paper determines the contribution of the impact air flow to the heat transfer during the cooling of the vertical surface.
\end{abstract}

\section{Introduction}

The development of modern power equipment is aimed at increasing their specific power, on the one hand, and reducing the size of the unit, on the other hand. With the increase in the power of small size electronic devices, the accumulation of heat leads to an increase in the operating temperature that affects the stability of the device and leads to a decrease in its life-time. Standard methods of single-phase convection do not meet the requirements for maintaining an acceptable operating temperature, compared to methods using latent heat of vaporization: boiling, evaporation, etc. Pulse cooling of dispersed liquid is a quick way to remove large heat fluxes having many advantages, such as for example, low coolant flow and high dissipation.

It is known that in order to realize effective cooling, it is necessary to ensure an even distribution of the mass concentration of the liquid not only on the surface of the heat exchanger, but also during the movement of the cooling mass [1]. The density of the heat flux will be the greater, the higher the mass concentration of the liquid in the air flow. The overwhelming number of works on cooling by means of gas-drop flows is devoted to various boiling regimes Well-studied modes of film cooling are widely used depending on the temperature of the cooled surface, its properties and geometry. In this paper we present data on the behavior of a liquid film formed on a large underheated surface by a pulsed gasdroplet flow.

\footnotetext{
* Corresponding author: Flags712008@yandex.ru
} 


\section{Experimental setup and procedure}

The experimental installation is a multifunctional stand consisting of the following units: a programmable multi-jet source of pulsed gas-droplet flow, an automated calorimeter, an automated system for recording the parameters of the flow, which includes original capacitive sensors for recording the thickness and velocity of the liquid film waves [2]. The controlled source of the gas-droplet jet is structurally designed as a two-chamber unit: for air and water. Flat part of the source is provided with 16 liquid injectors. The liquid injector is a sprayer of four nozzles with a diameter of $125 \mu \mathrm{m}$, connected by one solenoid valve. On this same surface, 25 gas nozzles with an outlet opening diameter of $0.5 \mathrm{~mm}$ are installed to create a gas-droplet air flow. The design of the multi-nozzle source was performed subject to the condition that the liquid nozzle is surrounded by 4 air nozzles, i.e. is in the "box". The distance between the source and the heat-loaded surface is $230 \mathrm{~mm}$.

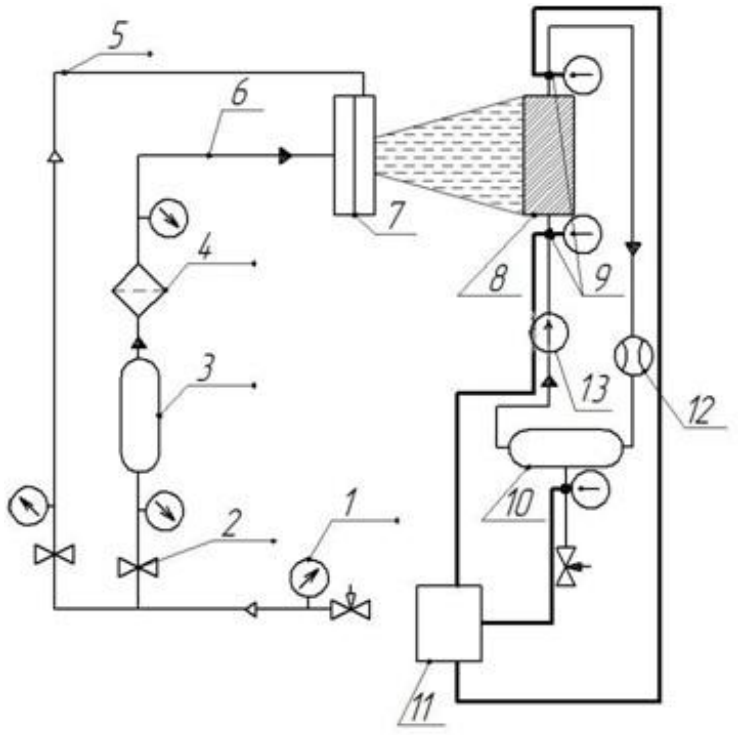

$$
\begin{aligned}
& 1 \text { - manometer } \\
& 2 \text { - valve } \\
& 3 \text { - liquid container } \\
& 4 \text { - filter } \\
& 5 \text { - air line } \\
& 6 \text { - water line } \\
& 7 \text { - injector unit } \\
& 8 \text { - effective area } \\
& 9 \text { - temperature sensor } \\
& 10 \text { - tank } \\
& 11 \text { - control block } \\
& 12 \text { - flowmeter } \\
& 13 \text { - pump } \\
& \text { - - elements of the pipeline } \\
& \text { - - elements of electrical system }
\end{aligned}
$$

Fig. 1. Schematic of experimental setup

The film flow registration system, built on video surveillance, high-speed photography and data from a multichannel dielkometric registrar on the local film thickness, made it possible to perform measurements of the induced flow on the surface of the heat exchanger. Figure 2 shows the general velocity map obtained from video surveillance data. In the flows displaced to the periphery, several different types of three-dimensional instabilities occur at low Reynolds numbers in different zones of the heat exchanger. Optical observations showed the existence of stable two- and three-dimensional waves of small amplitude $(\mathrm{dh} / \mathrm{h}$ $=0.05$ ) with transverse modulation along the flow outside the isolated regions $\mathrm{A}, \mathrm{D}, \mathrm{C}$, and D. Especially are highlighted the characteristic zones A, B, C, and D, where the measurements showed a weak current and the absence of large waves. 


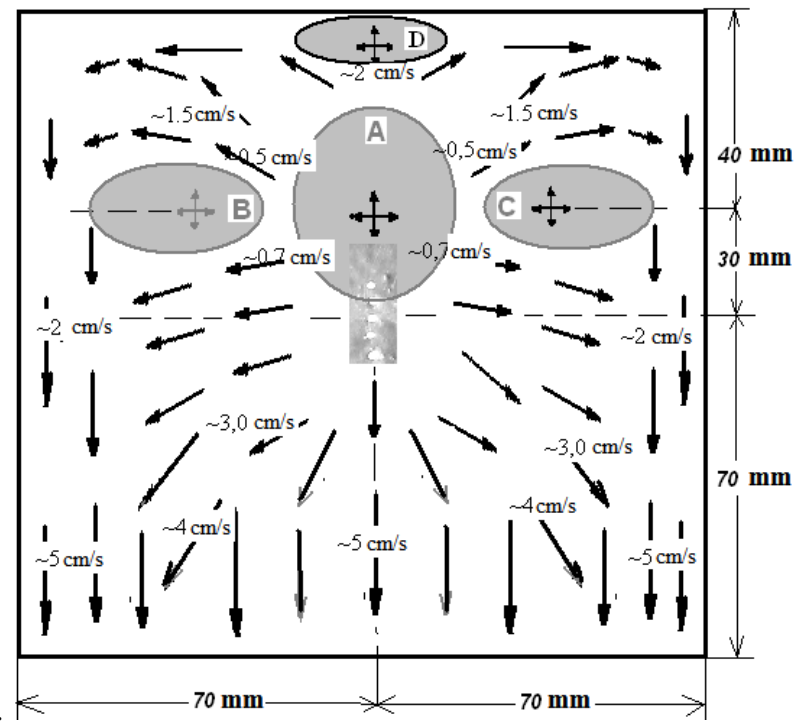

Fig. 2. Wave current projection map for cooling film

Figure 3 shows the film thickness variation $\left(\mathrm{h}_{\max }=0.92 \mathrm{~mm}\right)$ in zone A within $\mathrm{dh}=$ $0.06 \mathrm{~mm}$ on probe No. 1 and $\mathrm{dh}=0.02 \mathrm{~mm}$ on probe No. 2. In addition to slow waves, the recorder showed an induced pulsation, which can be attributed to the motion of very weak slow waves with an amplitude $\mathrm{dH}=0.06 \mathrm{~mm}$ from the center to the edges of the heat exchanger at a velocity $\mathrm{V}=(0-0.01) \mathrm{m} / \mathrm{s}, \mathrm{Re}=(4 \ldots 12)$ and the oscillation frequency $\mathrm{F}=$ $(0.2 \ldots 1) \mathrm{Hz}$.

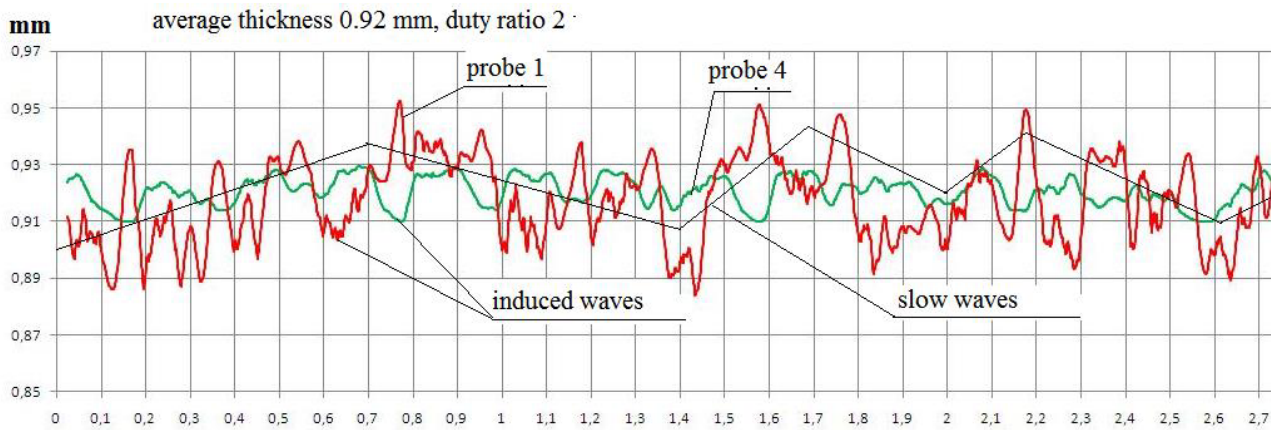

Fig. 3. Local film thickness

\section{Conclusions}

The performed studies have shown that when comparing the data on the structure and projections of the wall air flow velocities with the local flow of the film, we can conclude:

1. A wide multi-jet impact flow destroys the standard formation mechanism of a gravitational cooling film.

2. In the inner region under the impact flow, local stagnant zones of the cooling film are formed with induced pulsations.

3. The condition for the formation of these zones is the equality of multidirectional capillary and gravitational forces in the film as well as frictional forces from the "wall" streams of impact air jets on the film. 
4. In stagnant zones, provided that there is no film flow and active "turbulence" with drops, maximum heat transfer conditions are created due to convective and evaporative cooling.

5. The most energy-efficient cooling mode is the "single" pulse mode, where the heat exchanger surface is irrigated by an ensemble of drops.

The work was financially supported by RFBR (project No. 16-38-00853) and the grant of Russian President No. NSh-8780.2016.8.

\section{References}

1. A.L.N. Moreira, J. Carvalho, M.R.O. Panao, Int. J. Heat Fluid Flow 2007 28, 191 (2007)

2. A. Nazarov, A.Serov, M. Bodrov, Technical Phys. 80, 724 (2010) 